\title{
Increased Cardiovascular Risk in Patients with Adrenal Insufficiency: A Short Review
}

\author{
Amir-Hossein Rahvar, ${ }^{1}$ Christian S. Haas, ${ }^{2}$ Sven Danneberg, ${ }^{3}$ and Birgit Harbeck ${ }^{3}$ \\ ${ }^{1}$ Department of Medicine III, University of Hamburg, Hamburg, Germany \\ ${ }^{2}$ Department of Internal Medicine, University of Marburg, Marburg, Germany \\ ${ }^{3}$ Department of Medicine I, University of Luebeck, Luebeck, Germany \\ Correspondence should be addressed to Amir-Hossein Rahvar; a.rahvar@uke.de
}

Received 22 August 2017; Revised 6 November 2017; Accepted 15 November 2017; Published 10 December 2017

Academic Editor: Flavia Prodam

Copyright (C) 2017 Amir-Hossein Rahvar et al. This is an open access article distributed under the Creative Commons Attribution License, which permits unrestricted use, distribution, and reproduction in any medium, provided the original work is properly cited.

Cardiovascular disease (CVD) is the most common cause of death in the world. Recent studies have shown an association between adrenal insufficiency (AI) and increased cardiovascular risk (CVR). Patients with AI receive glucocorticoid (GC) replacement therapy which can lead to varying levels of blood cortisol. It was shown that these imbalances in blood cortisol may lead to a higher prevalence of coronary heart disease, major adverse coronary events, and increased mortality. GC substitution is essential in the treatment of AI without which the disease has been shown to be fatal. The most frequently used GC formula for replacement therapy is hydrocortisone (HC). There is no uniform opinion on hydrocortisone replacement therapy. Alternative GC such as prednisolone is also in use. Overreplacement of GC may lead to adverse effects including obesity, high blood pressure, and hyperglycaemia. Outcome may vary between primary and secondary AI mainly due to differences in the renin-angiotensin-aldosterone system (RAAS). Furthermore, decreased blood levels of cortisol may lead to a compensatory secretion of inflammatory mediators such as Interleukin-1 (IL-1), Interleukin-6 (IL-6), and/or tumor-necrosis factor (TNF). Physicians and patients should be properly educated about the increased risk of CVD in patients with AI.

\section{Adrenal Insufficiency: General Overview}

Primary adrenal insufficiency $(\mathrm{AI})$ is a rare disease with a prevalence of approximately 100 to 126 cases per million in Western countries [1-3]. Global data on AI prevalence however is sparse. While in developed countries the leading etiology of primary AI is autoimmune disease, tuberculosis associated adrenalitis remains an important cause in developing countries $[4,5]$. Certain drugs such as mitotane, ketoconazole, metyrapone, and etomidate may lead to primary adrenal insufficiency due to their inhibiting effect on adrenal enzymes [6]. ACTH-deficiency in the pituitary gland results in secondary AI with an estimated prevalence of 45.5 per 100,000 [7]. Both types of AI lead to a lack of endogenous glucocorticoids (GC), while absence of mineralocorticoids is limited to primary AI. Clinical manifestations of primary AI include fatigue, hypotension, hyponatremia, hyperkalemia, hypoglycaemia, and hyperpigmentation of the skin [8].
In comparison, clinical features of secondary AI exclude hypotension, hyperkalemia, and hyperpigmentation due to normal function of the renin-angiotensin-aldosterone system (RAAS) and low levels of ACTH. Hypoglycemia can occur more often in patients with central hypoadrenalism since growth-hormone deficiency may also be present [9]. GC replacement is necessary in both primary and secondary AI to prevent harmful symptoms or even death. The following review attempts to depict the cardiovascular risk (CVR) in patients with AI receiving GC replacement therapy.

\section{Glucocorticoid Replacement Therapy}

The first synthetic GC was discovered in 1949 by Hench et al. [10]. Nowadays, hydrocortisone (HC) is the most frequently used GC substitution formula in both primary and secondary AI. Of note, $\mathrm{HC}$ corresponds to endogenous cortisol in regard to bioavailability and receptor affinity [11]. During the last 
years, a once-daily oral HC dual-release tablet was developed [12]. Other common formulas include prednisolone and dexamethasone, although the use of the latter has been advised against by the current guideline on primary AI by the Endocrine Society [8]. A recent web-based survey by Forss et al. including 1,245 patients with AI showed that $3 / 4$ were using $\mathrm{HC}$ as replacement therapy [13]. The average dose of $\mathrm{HC}$ is 15-25 mg per day in both primary and secondary AI, divided into two or three doses $[8,14]$. Furthermore, fludrocortisone is given to patients with primary AI demonstrating low levels of aldosterone at a dose of $100 \mu \mathrm{g} / \mathrm{d}$ [8]. To mimic the physiological GC peak the highest dose of HC is usually taken early in the day. Nonetheless, all regimens used so far fail to exactly mirror the physiological circadian rhythm, thereby having a negative impact on the metabolic system. However, other approaches like using a four-dose regimen were not able to show significant changes in quality of life, body weight, blood pressure, or glucose levels compared to a two-dose regimen $[15,16]$. Recent studies showed an improvement in quality of life and certain metabolic parameters such as waist circumference, $\mathrm{HbAlc}$, and serum lipids after changing from hydrocortisone to a dual-release preparation (Plenadren, PLEN) [17]. Reduction of the $\mathrm{HC}$ dose by as much as $50 \%$ resulted in a significant weight loss of up to $7 \mathrm{~kg}$ in one year while blood pressure, fasting glucose, and insulin levels remained unaffected $[18,19]$. It remains unclear if the decrease in body weight is an expression of underreplacement or beneficial. In a study by Schulz et al. a reduction of HC doses resulted in higher bone mineral density analysed over the course of two years while no occurrence of adrenal crisis was mentioned [20]. Furthermore, monitoring the effectiveness of hydrocortisone replacement therapy seems to be a challenge. While urinary cortisol excretion has been shown to be helpful in the diagnostic approach to hypercortisolism, significant interindividual variations in both primary and secondary AI do not favour this method in hypocortisolism [21]. Salivary cortisol measurements have been in use for more than 50 years and may present a suitable monitoring parameter for the measurement of proper hydrocortisone replacement therapy for the future $[22,23]$.

In clinical practice, the objective of hydrocortisone replacement therapy is to keep doses as low as possible, while trying to avoid symptoms of $\mathrm{AI}$ and to minimize the risk of adrenal crisis. An appropriate increase of HC doses is necessary in situations of physical and mental stress. Thus, a proper education of AI patients and physicians on adapting $\mathrm{HC}$ dose in various scenarios is essential to improve patient outcome [25].

\section{Increased Cardiovascular Risk in Glucocorticoid Replacement Therapy}

Current GC replacement regimens try to mimic the physiological rhythm of endogenous cortisol as accurately as possible. However, temporary supra- and subphysiological levels of blood cortisol are common and may be harmful. In fact, patients with AI on GC replacement therapy appear to have an increased overall mortality [26, 27]. Recently, some studies have shown that this is mainly due to an increased cardiovascular risk (CVR) owing to the GC replacement therapy itself [28]. A dose of hydrocortisone exceeding $20 \mathrm{mg}$ per day seems to be associated with increased cardiovascular risk due to the higher prevalence of common metabolic risk factors [14]. It is conceivable that GC therapy affects some of the well-known risk factors for cardiovascular disease (CVD), for example, obesity, hypertension, diabetes, and hyperlipoproteinemia [26-29]. This can be observed in patients with Cushing's disease suffering from these symptoms due to an ACTH-producing tumor in the pituitary gland or a cortisol secreting tumor in the adrenal gland. Exogenous GC therapy may result in similar clinical manifestations that are also described as iatrogenic Cushing's disease. The ideal amount of GC substitution in AI patients remains at debate, especially when balancing advantages and adverse effects of the therapy.

In a population based case-control study with 50,656 patients having received at least one prescription for systemic or nonsystemic GCs, Souverein et al. showed an increased risk for cardiovascular and cerebrovascular events when compared to matching controls without GC intake with an adjusted odds-ratio of 1.25 (95\% confidence interval (CI) 1.21 to 1.29) (Table 1) [24].

A comparative study by Ross analysed lipid profiles in 110 AI patients from Sweden and South Africa (55 Swedish, 55 South African) regarding hydrocortisone doses [30]. Interestingly, the South African patients showed worse lipid profiles despite the use of lower HC doses. Therefore lipid levels may not solely depend on the chosen dose of $\mathrm{HC}$ but may also be affected by environmental factors and genetic predisposition [30].

Adipokine circulation showed no significant discrepancy when comparing healthy subjects with patients suffering from primary adrenal insufficiency receiving hydrocortisone replacement therapy, therefore suggesting no increased CVR in regard to lipid profiles [31]. Higher levels of low-density lipoprotein (LDL) were identified in patients receiving prednisolone instead of hydrocortisone while HbAlc, highdensity lipoprotein and triglyceride levels, body mass index, systolic and diastolic blood pressure, and waist circumference showed no significant difference [32]. Of interest, patients suffering from hormone-inactive tumors of the pituitary receiving hydrocortisone replacement therapy seem to have an increased risk of developing diseases such as hypertension, diabetes, hyperlipoproteinaemia, coronary heart disease, or atrial fibrillation when compared to those with normal hormone function or hormone producing adenomas of the pituitary [33]. In a study by Behan et al., lower doses of hydrocortisone in hypopituitary male patients showed an improved arterial stiffness index as well as a more physiological nocturnal dip in blood pressure [34]. This was in accordance with Petersons et al. (2014) who supposed that endothelial dysfunction contributes to the increased cardiovascular mortality associated with higher GC doses [35]. When comparing the prevalence of metabolic syndrome between male and female hypopituitary patients and healthy controls, Khang et al. showed an increased risk for female patients (39.8 versus $28.5 \%$ ) while the risk of male subjects appeared to be unaffected [36]. 
TABLE 1: Use of oral glucocorticoids (GC) and cardiovascular or cerebrovascular events, stratified according to type of outcome event among cases $(n=50656)$ and controls $(n=50656)$.

\begin{tabular}{|c|c|c|c|}
\hline Outcome event & $\begin{array}{l}\text { Ischaemic heart disease } \\
{[\text { OR 95\% CI }]^{*}}\end{array}$ & $\begin{array}{c}\text { Heart failure } \\
{[\text { OR 95\% CI }]^{*}}\end{array}$ & $\begin{array}{c}\text { Stroke/TIA } \\
{[\text { OR 95\% CI }]^{*}}\end{array}$ \\
\hline \multirow{2}{*}{ Ever used oral GC } & $5,298(25.6 \%)$ & $6,401(44.4 \%)$ & $3,656(23.4 \%)$ \\
\hline & [1.09 (1.03-1.15)] & [1.91 (1.79-2.03)] & {$[0.95(0.89-1.01)]$} \\
\hline \multicolumn{4}{|c|}{ Timing of oral GC use } \\
\hline \multirow{2}{*}{ Current use } & $2,507(12.1 \%)$ & $4,020(27.9 \%)$ & $1,640(10.5 \%)$ \\
\hline & [1.20 (1.11-1.29)] & {$[2.66(2.46-2.87)]$} & [0.91 (0.84-0.99)] \\
\hline \multirow{2}{*}{ Recent use } & $1310(6.3 \%)$ & $1,299(9.0 \%)$ & $882(5.7 \%)$ \\
\hline & [0.93 (0.85-1.02)] & [1.40 (1.27-1.55)] & {$[0.89(0.80-0.99)]$} \\
\hline \multirow{2}{*}{ Past use } & $1,481(7.2 \%)$ & $1,082(7.5 \%)$ & $1,134(7.3 \%)$ \\
\hline & {$[1.07(0.98-1.17)]$} & {$[1.19(1.08-1.32)]$} & {$[1.06(0.96-1.17)]$} \\
\hline
\end{tabular}

* Adjusted for the use of NSAIDs, hormone replacement therapy, antihypertensive drugs, nitrates, oral anticoagulants, antiplatelet drugs, antidiabetic drugs, bronchodilators, cromoglycates, inhaled glucocorticoids, DMARDs, smoking, and BMI, adapted to [24].

\section{Mineralocorticoid Replacement in Adrenal Insufficiency}

Primary adrenal insufficiency may include an absence of mineralocorticoids (MC) resulting in a need for daily substitution of fludrocortisone at a recommended dose of $100 \mu \mathrm{g} / \mathrm{d}$ [8]. Overreplacement may lead to arterial hypertension and electrolyte imbalances due to an overstimulation of the RAAS. Of note, MC secretion is subject to a circadian rhythm similar to that of GC [37].

Patients with secondary AI have a functioning RAAS. Therefore there is no need for mineralocorticoid substitution in patients with central hypoadrenalism. When studying the effects of GC therapy on the cardiovascular system these differences in mineralocorticoid effect of different steroids should be taken into consideration. Higher doses of hydrocortisone show an increase in blood pressure with lower levels of aldosterone and renin in patients with secondary AI [38]. Prednisolone, for example, shows lower affinity to the MC receptors than hydrocortisone while dexamethasone shows no MC effect at all [8].

\section{Inflammatory Markers}

Another conceivable option to study CVR in AI is temporary hypocortisolism and subsequent increase of inflammatory markers such as Interleukin 1 (IL-1), Interleukin 6 (IL-6), and tumor-necrosis factor (TNF). Mastorakos et al. showed a correlation between blood cortisol levels and these inflammatory mediators [39].

In another study by Papanicolaou et al. from 1996 IL1, IL-6, and TNF were measured multiple times in patients with Cushing's disease before and after surgery [40]. While blood cortisol levels were reduced to hypocortisolemic levels on the fourth and fifth postoperative day, a significant rise of circulating IL- 6 was seen. Postoperative elevations for IL-1 and TNF were also noted but to a lesser extent. Furthermore, subcutaneous injections of recombinant IL-6 at a dose of $3.0 \mu \mathrm{g} / \mathrm{kg}$ in healthy volunteers led to a significant reduction of corticosteroid-binding globulin and a rise of free blood cortisol $[41,42]$.
A collaborative meta-analysis from 2012 by Sarwar and Butterworth showed a slight risk reduction for coronary heart disease in patients with a genetic variant of the Interleukin-6 receptor [43]. These patients showed decreased levels of Creactive protein and fibrinogen due to lower stimulation of the IL- 6 inflammatory cascade. Blockage of the IL- 6 receptor using the monoclonal antibody tocilizumab in patients with rheumatoid arthritis appears to have a beneficial effect on coronary heart disease [44].

Overall, these studies indicate a correlation between cortisol and proinflammatory mediators such as IL-1, IL-6, and TNF, with these markers being associated with increased risk for cardiovascular events $[45,46]$.

\section{Conclusions}

While analysing past studies regarding the topic of cardiovascular risk in patients with adrenal insufficiency this reviewer recognizes a strong need for patient education in regard to not only the underlying disease itself but also a risk reduction of known factors such as obesity, high blood pressure, diabetes mellitus, and hyperlipidaemia. Patients should be informed about the increased risk for cardiovascular events and the need for regular check-ups, in the field of not only endocrinology but cardiology as well. Physicians in general but especially cardiologists should be educated about the higher incidence of cardiovascular events in these patients and recognize clinical manifestations of AI that may vary strongly between patients. Furthermore, GC replacement therapy in AI has not changed significantly over the past decades. While current replacement regimens try to mimic the physiological secretion of cortisol as accurately as possible, there is still a lot of room for improvement. Similar to insulin therapy in diabetes mellitus, GC replacement therapy should focus on avoiding serious side effects while guaranteeing an adequate substitution of the deficient hormone. New synthetic cortisol formulas introduced in recent years with a delayed-release may deliver better therapeutic options. Previous studies showed increased quality of life and improvement in metabolic profile using 
dual-release hydrocortisone preparations such as Plenadren. Furthermore, the slow-releasing mechanism of these new glucocorticoid substitution formulas may also avoid temporary subphysiological levels of cortisol. Further studies on this subject are required to improve patient care and outcome in adrenal insufficiency.

\section{Conflicts of Interest}

All authors declare that there are no conflicts of interest.

\section{References}

[1] A. C. Willis, "The prevalence of Addison's disease in Coventry, UK," Postgraduate Medical Journal, vol. 73, no. 859, pp. 286-288, 1997.

[2] S. Laureti, L. Vecchi, F. Santeusanio, and et al, "Is the prevalence of addison's disease underestimated?" The Journal of Clinical Endocrinology \& Metabolism, vol. 84, no. 5, 1999.

[3] G. Meyer, K. Badenhoop, and R. Linder, "Addison's disease with polyglandular autoimmunity carries a more than $2 \cdot 5$-fold risk for adrenal crises: German Health insurance data 2010-2013," Clinical Endocrinology, vol. 85, no. 3, pp. 347-353, 2016.

[4] M. M. Erichsen, K. Løvås, B. Skinningsrud et al., "Clinical, immunological, and genetic features of autoimmune primary adrenal insufficiency: observations from a Norwegian registry," The Journal of Clinical Endocrinology \& Metabolism, vol. 94, no. 12, pp. 4882-4890, 2009.

[5] Y.-K. Guo, Z.-G. Yang, Y. Li et al., "Addison's disease due to adrenal tuberculosis: Contrast-enhanced CT features and clinical duration correlation," European Journal of Radiology, vol. 62, no. 1, pp. 126-131, 2007.

[6] S. R. Bornstein, "Predisposing factors for adrenal insufficiency," The New England Journal of Medicine, vol. 360, no. 22, pp. 23282339, 2009.

[7] H. J. Schneider, G. Aimaretti, I. Kreitschmann-Andermahr, G.K. Stalla, and E. Ghigo, "Hypopituitarism," The Lancet, vol. 369, no. 9571, pp. 1461-1470, 2007.

[8] S. R. Bornstein, B. Allolio, W. Arlt et al., "Diagnosis and treatment of primary adrenal insufficiency: an endocrine society clinical practice guideline," The Journal of Clinical Endocrinology \& Metabolism, vol. 101, no. 2, pp. 364-389, 2016.

[9] B. Bleicken, S. Hahner, M. Ventz, and M. Quinkler, "Delayed diagnosis of adrenal insufficiency is common: A cross-sectional study in 216 patients," The American Journal of the Medical Sciences, vol. 339, no. 6, pp. 525-531, 2010.

[10] P. S. Hench, E. C. Kendall, C. H. Slocumb, and H. F. Polley, "Adrenocortical hormone in arthritis: Preliminary report," Annals of the Rheumatic Diseases, vol. 8, no. 2, pp. 97-104, 1949.

[11] H. Derendorf, H. Möllmann, J. Barth, C. Möllmann, S. Tunn, and M. Krieg, "Pharmacokinetics and Oral Bioavailability of Hydrocortisone," The Journal of Clinical Pharmacology, vol. 31, no. 5, pp. 473-476, 1991.

[12] G. Johannsson, AG. Nilsson, R. Bergthorsdottir et al., "Improved cortisol exposure-time profile and outcome in patients with adrenal insufficiency: a prospective randomized trial of a novel hydrocortisone dual-release formulation," The Journal of Clinical Endocrinology and Metabolism, vol. 97, no. 2, pp. 473-481, Feb 2012.
[13] M. Forss, G. Batcheller, S. Skrtic, and G. Johannsson, "Current practice of glucocorticoid replacement therapy and patientperceived health outcomes in adrenal insufficiency - a worldwide patient survey," BMC Endocrine Disorders, vol. 12, article no. $8,2012$.

[14] H. Filipsson, J. P. Monson, M. Koltowska-Häggström, A. Mattsson, and G. Johannsson, "The impact of glucocorticoid replacement regimens on metabolic outcome and comorbidity in hypopituitary patients," The Journal of Clinical Endocrinology \& Metabolism, vol. 91, no. 10, pp. 3954-3961, 2006.

[15] B. Ekman, M. Bachrach-Lindström, T. Lindström, J. Wahlberg, J. Blomgren, and H. J. Arnqvist, "A randomized, double-blind, crossover study comparing two- and four-dose hydrocortisone regimen with regard to quality of life, cortisol and $\mathrm{ACTH}$ profiles in patients with primary adrenal insufficiency," Clinical Endocrinology, vol. 77, no. 1, pp. 18-25, 2012.

[16] B. Bleicken, S. Hahner, M. Loeffler et al., "Influence of hydrocortisone dosage scheme on health-related quality of life in patients with adrenal insufficiency," Clinical Endocrinology, vol. 72, no. 3, pp. 297-304, 2010.

[17] R. Giordano, F. Guaraldi, E. Marinazzo et al., "Improvement of anthropometric and metabolic parameters, and quality of life following treatment with dual-release hydrocortisone in patients with Addison's disease," Endocrine Journal, vol. 51, no. 2, pp. 360-368, 2016.

[18] F. P. Dunne, P. Elliot, M. D. Gammage et al., "Cardiovascular junction and glucocorticoid replacement in patients with hypopituitarism," Clinical Endocrinology, vol. 43, no. 5, pp. 623629, 1995.

[19] K. Danilowicz, O. D. Bruno, M. Manavela, R. M. Gomez, and A. Barkan, "Correction of cortisol overreplacement ameliorates morbidities in patients with hypopituitarism: A pilot study," The Pituitary Society, vol. 11, no. 3, pp. 279-285, 2008.

[20] J. Schulz, K. R. Frey, M. S. Cooper et al., "Reduction in daily hydrocortisone dose improves bone health in primary adrenal insufficiency," European Journal of Endocrinology, vol. 174, no. 4, pp. 531-538, 2016.

[21] C. S. Haas, A.-H. Rahvar, S. Danneberg, H. Lehnert, H. Moenig, and B. Harbeck, "Low Impact of Urinary Cortisol in the Assessment of Hydrocortisone Replacement Therapy," Hormone and Metabolic Research, vol. 48, no. 9, pp. 571-574, 2016.

[22] W. J. Inder, G. Dimeski, and A. Russell, "Measurement of salivary cortisol in 2012 - Laboratory techniques and clinical indications," Clinical Endocrinology, vol. 77, no. 5, pp. 645-651, 2012.

[23] H. Raff, "Utility of salivary cortisol measurements in Cushing's syndrome and adrenal insufficiency," The Journal of Clinical Endocrinology \& Metabolism, vol. 94, no. 10, pp. 3647-3655, 2009.

[24] P. C. Souverein, A. Berard, T. P. Van Staa et al., "Use of oral glucocorticoids and risk of cardiovascular and cerebrovascular disease in a population based case-control study," Heart, vol. 90, no. 8, pp. 859-865, 2004.

[25] D. Kampmeyer, H. Lehnert, H. Moenig, C. S. Haas, and B. Harbeck, "A strong need for improving the education of physicians on glucocorticoid replacement treatment in adrenal insufficiency: An interdisciplinary and multicentre evaluation," European Journal of Internal Medicine, vol. 33, pp. e13-e15, 2016.

[26] K. Krzyzanowska, C. Schnack, F. Mittermayer et al., "High prevalence of abnormal circadian blood pressure regulation and impaired glucose tolerance in adults with hypopituitarism," 
Experimental and Clinical Endocrinology \& Diabetes, vol. 113, no. 8, pp. 430-434, 2005.

[27] E. M. Erfurth and L. Hagmar, "Cerebrovascular disease in patients with pituitary tumors," Trends in Endocrinology \& Metabolism, vol. 16, no. 7, pp. 334-342, 2005.

[28] M. Debono, R. J. Ross, and J. Newell-Price, "Inadequacies of glucocorticoid replacement and improvements by physiological circadian therapy," European Journal of Endocrinology, vol. 160, no. 5, pp. 719-729, 2009.

[29] M. Terzolo, A. Pia, A. Alì et al., "Adrenal incidentaloma: a new cause of the metabolic syndrome?" The Journal of Clinical Endocrinology \& Metabolism, vol. 87, no. 3, pp. 998-1003, 2002.

[30] I. L. O. Ross, "Cardiovascular risk factors in patients with Addison's disease: a comparative study of South African and Swedish patients," PLoS ONE, vol. 9, no. 6, p. e90768, 2014.

[31] M. Fichna, P. Fichna, M. Gryczyńska, A. Czarnywojtek, M. Zurawek, and M. Ruchała, "Steroid replacement in primary adrenal failure does not appear to affect circulating adipokines," Endocrine Journal, vol. 48, no. 2, pp. 677-685, 2015.

[32] M. Quinkler, B. Ekman, C. Marelli, S. Uddin, P. Zelissen, and R. D. Murray, "Prednisolone is associated with a worse lipid profile than hydrocortisone in patients with adrenal insufficiency," Endocrine Connections, vol. 6, no. 1, pp. 1-8, 2016.

[33] B. Harbeck, C. S. Haas, S. Suefke, and H. Moenig, "Cardiovascular risk factors and disease in patients with hypothalamicpituitary disorders," International Journal of Cardiology, vol. 184, no. 1, pp. 464-465, 2015.

[34] L.-A. Behan, D. Carmody, B. Rogers et al., "Low-dose hydrocortisone replacement is associated with improved arterial stiffness index and blood pressure dynamics in severely adrenocorticotrophin-deficient hypopituitary male patients," European Journal of Endocrinology, vol. 174, no. 6, pp. 791-799, 2016.

[35] C. J. Petersons, B. L. Mangelsdorf, C. H. Thompson, and M. G. Burt, "Acute effect of increasing glucocorticoid replacement dose on cardiovascular risk and insulin sensitivity in patients with adrenocorticotrophin deficiency," The Journal of Clinical Endocrinology \& Metabolism, vol. 99, no. 6, pp. 2269-2276, 2014.

[36] A. R. Khang, E. J. Ku, Y. A. Kim et al., "Sex differences in the prevalence of metabolic syndrome and its components in hypopituitary patients: comparison with an age- and sexmatched nationwide control group," The Pituitary Society, vol. 19, no. 6, pp. 573-581, 2016.

[37] G. H. Williams, J. P. Cain, R. G. Dluhy, and R. H. Underwood, "Studies of the control of plasma aldosterone concentration in normal man. I. Response to posture, acute and chronic volume depletion, and sodium loading.", The Journal of Clinical Investigation, vol. 51, no. 7, pp. 1731-1742, 1972.

[38] J. W. Buning, M. Van Faassen, P. Brummelman et al., "Effects of hydrocortisone on the regulation of blood pressure: Results from a randomized controlled trial," The Journal of Clinical Endocrinology \& Metabolism, vol. 101, no. 10, pp. 3691-3699, 2016.

[39] G. Mastorakos, G. Paltoglou, M. Greene et al., "Inappropriately normal plasma ACTH and cortisol concentrations in the face of increased circulating interleukin- 6 concentration in exercise in patients with sarcoidosis," Stress, vol. 16, no. 2, pp. 202-210, 2013.

[40] D. A. Papanicolaou, C. Tsigos, E. H. Oldfield, and G. P. Chrousos, "Acute glucocorticoid deficiency is associated with plasma elevations of interleukin-6: Does the latter participate in the symptomatology of the steroid withdrawal syndrome and adrenal insufficiency?" The Journal of Clinical Endocrinology \& Metabolism, vol. 81, no. 6, pp. 2303-2306, 1996.

[41] C. Tsigos and I. Kyrou, "Prolonged suppression of corticosteroid-binding globulin by recombinant human interleukin-6 in man [3]," The Journal of Clinical Endocrinology \& Metabolism, vol. 83, no. 9, p. 3379, 1998.

[42] G. Mastorakos, G. P. Chrousos, and J. S. Weber, "Recombinant interleukin-6 activates the hypothalamic-pituitary-adrenal axis in humans," The Journal of Clinical Endocrinology \& Metabolism, vol. 77, no. 6, pp. 1690-1694, 1993.

[43] N. Sarwar and A. S. Butterworth, "Interleukin-6 receptor pathways in coronary heart disease: a collaborative meta-analysis of 82 studies," The Lancet, vol. 379, no. 9822, pp. 1205-1213, 2012.

[44] D. I. Swerdlow, M. V. Holmes, K. B. Kuchenbaecker et al., "The interleukin-6 receptor as a target for prevention of coronary heart disease: a mendelian randomisation analysis," Lancet, vol. 379, no. 9822, pp. 1214-1224, 2012.

[45] J. Nilsson, "Cytokines and smooth muscle cells in atherosclerosis," Cardiovascular Research, vol. 27, no. 7, pp. 1184-1190, 1993.

[46] B. Vicenová, V. Vopálenský, L. Burýsek et al., "Emerging role of interleukin-1 in cardiovascular diseases," Physiological Research, vol. 58, no. 4, pp. 481-498, 2009. 


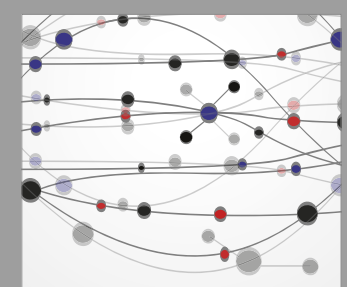

The Scientific World Journal
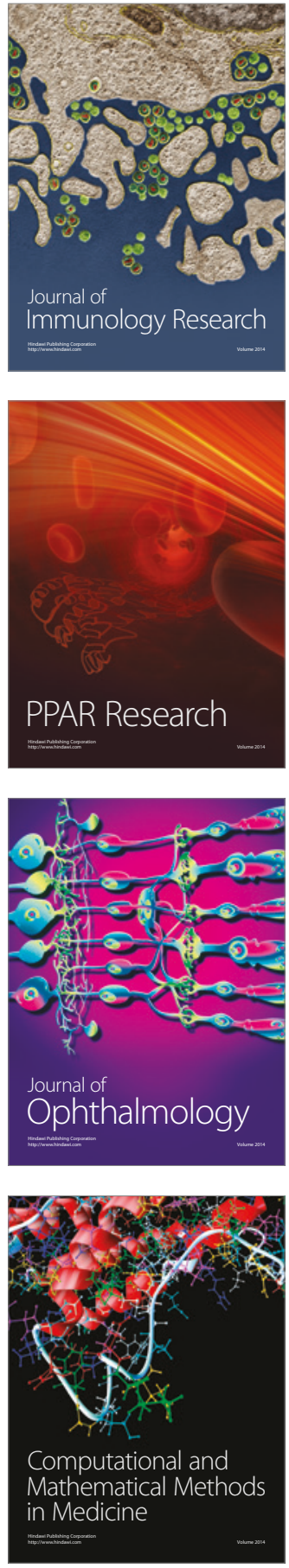

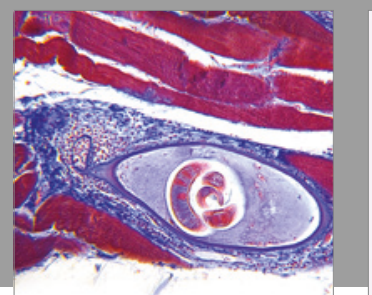

Gastroenterology Research and Practice
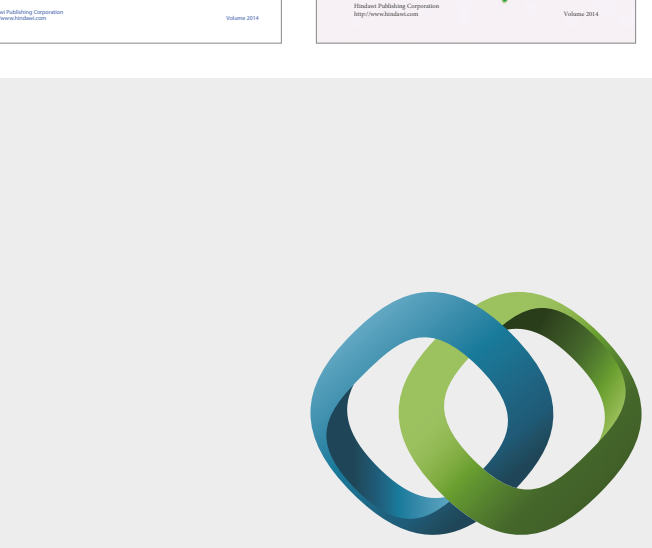

\section{Hindawi}

Submit your manuscripts at

https://www.hindawi.com
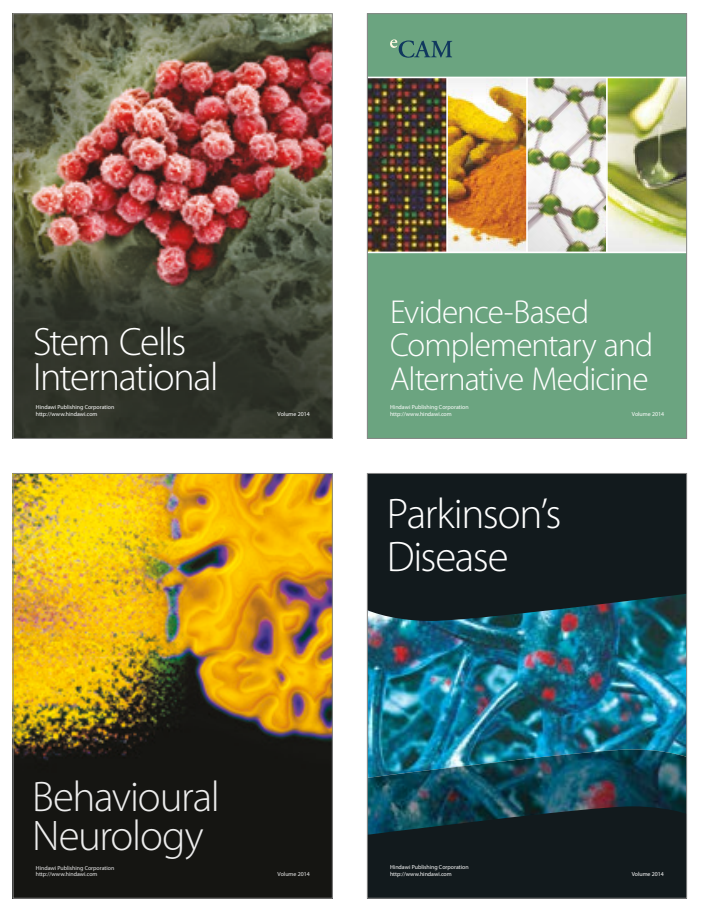
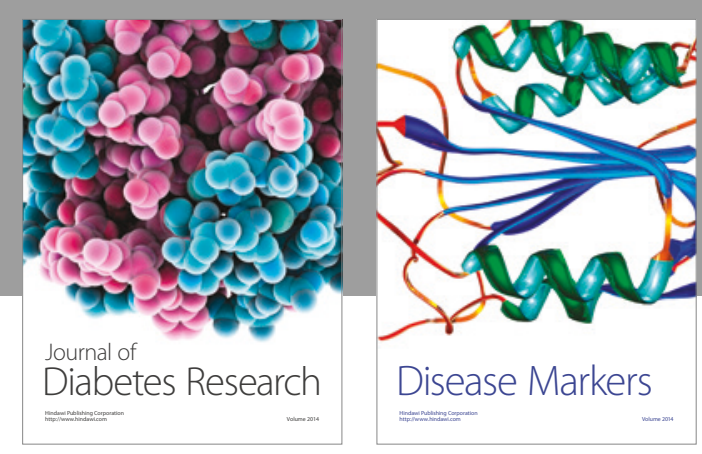

Disease Markers
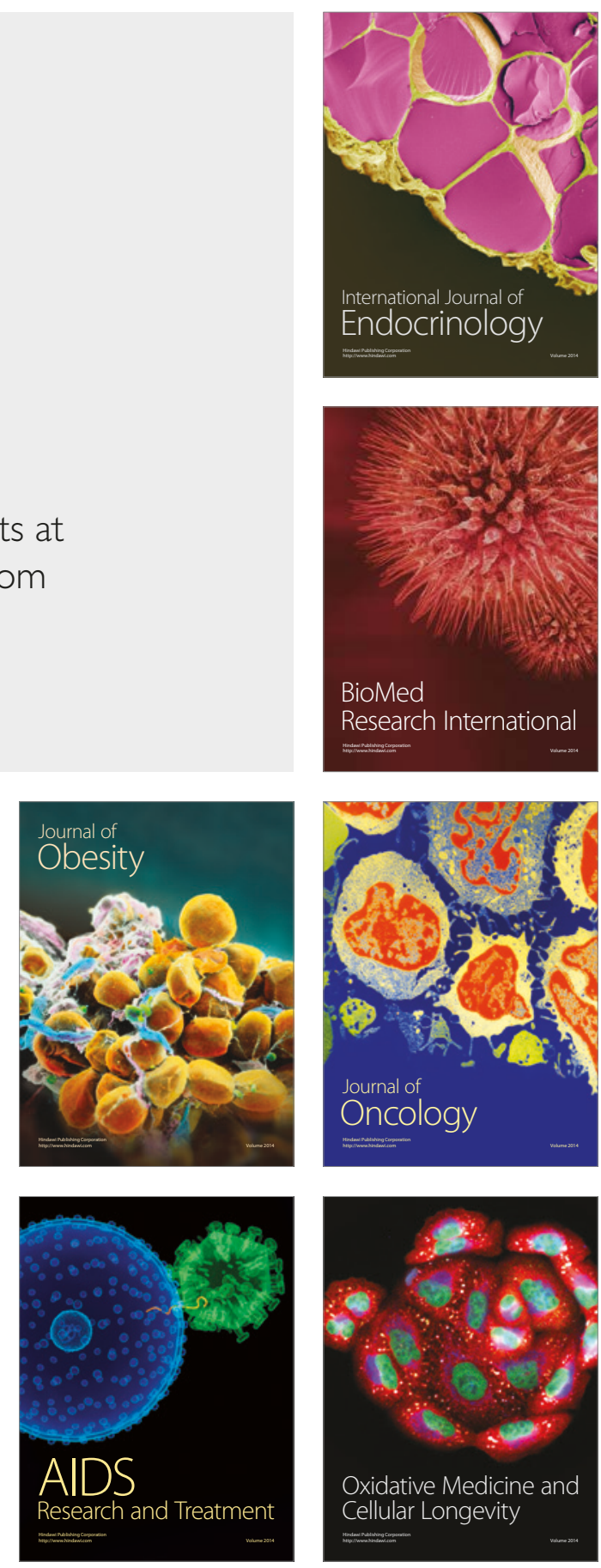